\title{
Comments on "What's in a relationship? On distinguishing property holding and object binding" by S. Kelly
}

\author{
H. C. Mayr \\ Dept. of Informatics, University of Klagenfurt \\ Universitätsstraße 65-67, A-9022 Klagenfurt, Austria \\ Phone:+43-463-2700579,E-mail:mayr@ifi.uni-klu.ac.at
}

Though the paper does not bring up really new concepts and ideas, it is, in my opinion, an important contribution to the conference's aim 'towards a consolidation of views'. I think so because of the somewhat ambivalent use of the notion of 'relationship' in modern approaches to conceptual design, i.e., methods for object-oriented analysis (OOA). Whereas in classical dataoriented conceptual (semantical) models like Entity-Relationship, NIAM etc., 'relationship' is a well-defined and well-understood concept, OOA methods

- either propose to describe relationships between objects by references on the basis of objectvalued attributes, corresponding to the situation on the level of logical object-oriented database models,

- or they provide for a relationship concept, but do so half-heartedly by, e.g., an unnatural restriction to binary relationships or by strongly recommending to replace $n$-ary relationships by binary ones.

However, if one considers, as I do, the essence of object orientation to consist of a structured system theory based view on universes of discourse, then it becomes evident, that the concepts of (n-ary) relationship and role are (helpful) semantical categories at their own: systems are composed of things, things either are objects that possibly are capable to actions or they are relationships between such objects. Things, and consequently, relationships may have properties, and in addition, relationships consist of roles binding objects. Roles have properties like cardinalities, optionality etc.

Thus it is adequate, as Kelly does, to distinguish between two different aspects of relationships, viz., being things, to hold properties, and, being relationships, to bind objects via component rules. It is the merit of the paper to revoke these aspects into attention. However, the presentation could have been somewhat more formally sound, e.g., the notion of 'rule' is not really clear (is it a consistency constraint, what are possible kinds of rules etc.?). Also, there is some confusion between the levels of arguments, i.e. metamodel level (concerning semantical power) and case tool/implementation level (concerning efficiency). In my opinion, the design of a metamodel should not be influenced by implementational aspects. In addition, semantical arguments should mainly focus on the shape of the final design results and not on intermediate ones.

Altogether, however, the paper has its merits as a valuable contribution to the domain of metamodelling with relationships. 